\title{
Viscosity Approximation Methods and Strong Convergence Theorems for the Fixed Point of Pseudocontractive and Monotone Mappings in Banach Spaces
}

\author{
Yan Tang \\ College of Mathematics and Statistics, Chongqing Technology and Business University, Chongqing 400067, China \\ Correspondence should be addressed to Yan Tang; ttyy7999@163.com
}

Received 22 January 2013; Revised 26 May 2013; Accepted 27 May 2013

Academic Editor: Filomena Cianciaruso

Copyright (C) 2013 Yan Tang. This is an open access article distributed under the Creative Commons Attribution License, which permits unrestricted use, distribution, and reproduction in any medium, provided the original work is properly cited.

\begin{abstract}
Suppose that $C$ is a nonempty closed convex subset of a real reflexive Banach space $E$ which has a uniformly Gateaux differentiable norm. A viscosity iterative process is constructed in this paper. A strong convergence theorem is proved for a common element of the set of fixed points of a finite family of pseudocontractive mappings and the set of solutions of a finite family of monotone mappings. And the common element is the unique solution of certain variational inequality. The results presented in this paper extend most of the results that have been proposed for this class of nonlinear mappings.
\end{abstract}

\section{Introduction}

Let $E$ be a real Banach space with dual $E^{*}$. A normalized duality mapping $J: E \rightarrow 2^{E^{*}}$ is defined by

$$
J x=\left\{f^{*} \in E^{*}:\left\langle x, f^{*}\right\rangle=\|x\|^{2}=\left\|f^{*}\right\|^{2}\right\},
$$

where $\langle\cdot, \cdot\rangle$ denotes the generalized duality pairing between $E$ and $E^{*}$. It is well known that $E$ is smooth if and only if $J$ is single valued, and if $E$ is uniformly smooth, then $J$ is uniformly continuous on bounded subsets of $E$. Moreover, if $E$ is reflexive and strictly convex Banach space with a strictly convex dual then $J^{-1}$ is single valued, one-to-one surjective, and it is the duality mapping from $E^{*}$ into $E$, and then $J J^{-1}=$ $I_{E^{*}}$ and $J J^{-1}=I_{E}$.

A mapping $A: D(A) \subset E \rightarrow E^{*}$ is said to be monotone if, for each $x, y \in D(A)$, the following inequality holds:

$$
\langle x-y, A x-A y\rangle \geq 0 \text {. }
$$

A mapping $A: D(A) \subset E \rightarrow E^{*}$ is said to be strongly monotone, if there exists a positive real number $\alpha>0$ such that for all $x, y \in D(A)$

$$
\langle x-y, A x-A y\rangle \geq \alpha\|A x-A y\|^{2} .
$$

Obviously, the class of monotone mappings includes the class of the $\alpha$-inverse strongly monotone mappings.

Let $C$ be closed convex subset of Banach space $E$. A mapping $T$ is said to be pseudocontractive if, for any $x, y \in$ $D(T)$, there exists $j(x-y) \in J(x-y)$ such that

$$
\langle T x-T y, j(x-y)\rangle \leq\|x-y\|^{2} .
$$

A mapping $T$ is said to be $\kappa$-strictly pseudo-contractive, if, for any $x, y \in D(T)$, there exist $j(x-y) \in J(x-y)$ and a constant $0 \leq \kappa \leq 1$ such that

$$
\langle x-y-(T x-T y), j(x-y)\rangle \geq \kappa\|(I-T) x-(I-T) y\|^{2} .
$$

A mapping $T: C \rightarrow C$ is called nonexpansive if

$$
\|T x-T y\| \leq\|x-y\|, \quad \forall x, y \in C .
$$

Clearly, the class of pseudo-contractive mappings includes the class of strict pseudo-contractive mappings and non-expansive mappings. We denote by $F(T)$ the set of fixed points of $T$; that is, $F(T)=\{x \in C: T x=x\}$.

A mapping $f: C \rightarrow C$ is called contractive with a contraction coefficient if there exists a constant $\rho \in(0,1)$ such that

$$
\|f(x)-f(y)\| \leq \rho\|x-y\|, \quad \forall x, y \in C .
$$


Let $E$ be a real Banach space with dual $E^{*}$. The norm on $E$ is said to be uniformly Gateaux differentiable if for each $y \in$ $S_{1}(0):=\{x \in E:\|x\|=1\}$ the $\operatorname{limit}_{\lim _{t \rightarrow 0}}(\|x+t y\|-\|x\|) / t$ exists uniformly for $x \in S_{1}(0)$.

For finding an element of the set of fixed points of the non-expansive mappings, Halpern [1] was the first to study the convergence of the scheme in 1967:

$$
x_{n+1}=\alpha_{n+1} u+\left(1-\alpha_{n+1}\right) T\left(x_{n}\right) .
$$

Viscosity approximation methods are very important because they are applied to convex optimization, linear programming, monotone inclusions, and elliptic differential equations. In Hilbert spaces, many authors have studied the fixed points problems of the fixed points for the non-expansive mappings and monotone mappings by the viscosity approximation methods, and obtained a series of good results (see [2-17]).

In 2000, Moudifi [18] introduced the viscosity approximation methods and proved the strong convergence of the following iterative algorithm in Hilbert spaces under some suitable conditions:

$$
x_{n+1}=\alpha_{n} f\left(x_{n}\right)+\left(1-\alpha_{n}\right) T\left(x_{n}\right) .
$$

Suppose that $A$ is monotone mapping from $C$ into $E$. The classical variational inequality problem is formulated as finding a point $u \in C$ such that $\langle v-u, A u\rangle \geq 0$, for all $v \in C$. The set of solutions of variational inequality problems is denoted by $\operatorname{VI}(C, A)$.

Takahashi and Toyoda $[19,20]$ introduced the following scheme in Hilbert spaces and studied the weak and strong convergence theorem of the elements of $F(T) \cap V I(C, A)$, respectively, under different conditions:

$$
x_{n+1}=\alpha_{n} x_{n}+\left(1-\alpha_{n+1}\right) T P_{C}\left(x_{n}-\lambda_{n} x_{n}\right),
$$

where $T$ is non-expansive mapping and $A$ is $\alpha$-inverse strong monotone operator.

Recently, Zegeye and Shahzad [21] introduced the following algorithm and obtained the strong convergence theorem but still in Hilbert spaces:

$$
x_{n+1}=\alpha_{n} f\left(x_{n}\right)+\left(1-\alpha_{n}\right) T_{r_{n}} F_{r_{n}} x_{n},
$$

where $T_{r_{n}}, F_{r_{n}}$ are nonexpansive mappings.

Our concern now is the following: is it possible to construct a new sequence in Banach spaces which converges strongly to a common element of fixed points of a finite family of pseudocontractive mappings and the solution set of a variational inequality problems for finite family of monotone mappings?

\section{Preliminaries}

In the sequel, we will use the following lemmas.

Lemma 1 (see, e.g., [5]). Let $\left\{a_{n}\right\}$ be a sequence of nonnegative real numbers satisfying the following relation:

$$
a_{n+1} \leq\left(1-\theta_{n}\right) a_{n}+\sigma_{n}, \quad n \geq 0,
$$

where $\left\{\theta_{n}\right\}$ is a sequence in $(0,1)$ and $\left\{\sigma_{n}\right\}$ is a real sequence such that

(i) $\sum_{n=0}^{\infty} \theta_{n}=\infty$;

(ii) $\lim \sup _{n \rightarrow \infty} \sigma_{n} / \theta_{n} \leq 0$ or $\sum_{n=0}^{\infty} \sigma_{n}<\infty$.

Then $\lim _{n \rightarrow \infty} a_{n}=0$.

Lemma 2 (see, e.g., [10]). Let $C$ be a nonempty, closed, and convex subset of uniformly smooth strictly convex real Banach space $E$ with dual $E^{*}$. Let $A: C \rightarrow E^{*}$ be a continuous monotone mapping; define mapping $F_{r}$ as follows: $x \in E, r \in(0, \infty)$

$$
\begin{gathered}
F_{r}(x)=\left\{z \in C:\langle y-z, A z\rangle+\frac{1}{r}\langle y-z, J z-J x\rangle \geq 0,\right. \\
\forall y \in C\} .
\end{gathered}
$$

Then the following hold:

(i) $F_{r}$ is well defined and single valued;

(ii) $F_{r}$ is a firmly non-expansive mapping; that is, $\left\langle F_{r} x-F_{r} y, J F_{r} x-J F_{r} y\right\rangle \leq\left\langle F_{r} x-F_{r} y, J x-J y\right\rangle ;$

(iii) $F\left(F_{r}\right)=V I(C, A)$;

(iv) $\operatorname{VI}(C, A)$ is closed and convex.

Lemma 3 (see, e.g., [22]). Let $C$ be a nonempty closed convex subset of uniformly smooth strictly convex real Banach space E. Let $T: C \rightarrow E$, be a continuous strictly pseudocontractive mapping; define mapping $T_{r}$ as follows: $x \in E, r \in(0, \infty)$

$T_{r}(x)$

$$
\begin{gathered}
=\left\{z \in C:\langle y-z, T z\rangle-\frac{1}{r}\langle y-z,(1+r) J z-J x\rangle \leq 0,\right. \\
\forall y \in C\} .
\end{gathered}
$$

Then the following hold:

(i) $T_{r}$ is single valued;

(ii) $T_{r}$ is a firmly nonexpansive mapping; that is,

$$
\left\langle T_{r} x-T_{r} y, J T_{r} x-J T_{r} y\right\rangle \leq\left\langle T_{r} x-T_{r} y, J x-J y\right\rangle ;
$$

(iii) $F\left(T_{r}\right)=F(T)$;

(iv) $F(T)$ is closed and convex.

Lemma 4 (see, e.g., [23]). Let $\left\{x_{n}\right\}$ and $\left\{z_{n}\right\}$ be bounded sequence in a Banach space, and let $\left\{\beta_{n}\right\}$ be a sequence in $[0,1]$ which satisfies the following condition:

$$
0<\liminf _{n \rightarrow \infty} \beta_{n}<\limsup _{n \rightarrow \infty} \beta_{n}<1 .
$$

Suppose

$$
\begin{gathered}
x_{n+1}=\beta_{n} x_{n}+\left(1-\beta_{n}\right) z_{n}, \quad n \geq 0, \\
\lim _{n \rightarrow \infty}\left(\left\|z_{n+1}-z_{n}\right\|-\left\|x_{n+1}-x_{n}\right\|\right) \leq 0 .
\end{gathered}
$$

Then $\lim _{n \rightarrow \infty}\left\|z_{n}-x_{n}\right\|=0$. 
Lemma 5 (see, e.g., [16]). Let $C$ be a nonempty closed and convex subset of a real smooth Banach space E. A mapping $\Pi_{C}: E \rightarrow C$ is a generalized projection. Let $x \in E$; then $x_{0}=\Pi_{C} x$ if and only if

$$
\left\langle z-x_{0}, J x-J x_{0}\right\rangle \leq 0, \quad \forall z \in C .
$$

Lemma 6. Let $E$ be a real Banach space with dual $E^{*} . J: E \rightarrow$ $2^{E^{*}}$ is the generalized duality pairing; then, for all $x, y \in E$,

$$
\|x+y\|^{2} \leq\|x\|^{2}+2\langle y, j(x+y)\rangle, \quad \forall j(x+y) \in J(x+y) .
$$

\section{Main Results}

Let $C$ be a nonempty closed convex and bounded subset of a smooth, strictly convex and reflexive real Banach space $E$ with dual $E^{*}$. Let $A_{i}: C \rightarrow E^{*}, i=1,2, \ldots, m$ be a finite family of continuous monotone mappings, and let $T_{i}: C \rightarrow$ $C, i=1,2, \ldots, m$, be a finite family of continuous strictly pseudo-contractive mappings. For the rest of this paper, $T_{i r_{n}} x$ and $F_{i r_{n}} x$ are mappings defined as follows: for $x \in E, r_{n} \in$ $(0, \infty)$,

$$
\begin{aligned}
& T_{i r_{n}}(x) \\
& :=\left\{z \in C:\left\langle y-z, T_{i} z\right\rangle-\frac{1}{r_{n}}\left\langle y-z,\left(1+r_{n}\right) J z-J x\right\rangle \leq 0,\right. \\
& \quad \forall y \in C\} .
\end{aligned}
$$

Consider

$$
\begin{aligned}
& F_{i r_{n}}(x) \\
& :=\left\{z \in C:\left\langle y-z, A_{i} z\right\rangle+\frac{1}{r_{n}}\langle y-z, J z-J x\rangle \geq 0,\right. \\
& \quad \forall y \in C\} .
\end{aligned}
$$

Denote $F_{1}=\cap_{i=1}^{m} F\left(T_{i r_{n}}\right), F_{2}=\cap_{i=1}^{m} F\left(F_{i r_{n}}\right)$.

Lemma 7. Let $C$ be a nonempty closed convex and bounded subset of a smooth Banach space E, and let $\Gamma_{i}: C \rightarrow C, i=$ $1,2, \ldots, m$, be a finite family of non-expansive mappings such that $\cap_{i=1}^{m} F\left(\Gamma_{i}\right) \neq \emptyset$. Suppose that $\alpha=\inf \left\{\alpha_{i}\right\}>0$; then there exists non-expansive mapping $\Gamma: C \rightarrow C$ such that $F(\Gamma)=$ $\cap_{i=1}^{m} F\left(\Gamma_{i}\right)$.

Proof. Let $\left\{\alpha_{i}\right\}$ be any sequence of positive real numbers satisfying $\sum_{i=1}^{m} \alpha_{i}=1$ and set $\Gamma=\sum_{i=1}^{m} \alpha_{i} \Gamma_{i}$. Since each $\Gamma_{i}$ is non-expansive for any $i \in\{1,2, \ldots, m\}$, we have that $\Gamma$ is well-defined nonexpansive mapping (see, e.g., [20]), and

$$
\begin{aligned}
\|\Gamma x-\Gamma y\| & =\left\|\sum_{i=1}^{m} \alpha_{i} \Gamma_{i} x-\sum_{i=1}^{m} \alpha_{i} \Gamma_{i} y\right\| \\
& \leq \sum_{i=1}^{m} \alpha_{i}\left\|\Gamma_{i} x-\Gamma_{i} y\right\| \leq\|x-y\| .
\end{aligned}
$$

Next, we claim that $F(\Gamma)=\cap_{i=1}^{m} F\left(\Gamma_{i}\right)$.
Clearly $\cap_{i=1}^{m} F\left(\Gamma_{i}\right) \subset F(\Gamma)$. Now, we prove that $F(\Gamma) \subset$ $\cap_{i=1}^{m} F\left(\Gamma_{i}\right)$. Let $x \in F(\Gamma)$ and $p \in \cap_{i=1}^{m} F\left(\Gamma_{i}\right)$. Then

$$
\begin{aligned}
0 & =\langle\Gamma x-x, j(x-p)\rangle=\left\langle\sum_{i=1}^{m} \alpha_{i} \Gamma_{i} x-x, j(x-p)\right\rangle \\
& =\sum_{i=1}^{m} \alpha_{i}\left\langle\Gamma_{i} x-x, j(x-p)\right\rangle
\end{aligned}
$$

and non-expansivity of each $\Gamma_{i}$ implies that $\left\langle\Gamma_{i} x-x, j(x-p)\right\rangle=$ 0 for each $i=1,2, \ldots, m$, which implies $\Gamma_{i} x=x$ for each $i=1,2, \ldots, m$. Therefore, $x \in \cap_{i=1}^{m} F\left(\Gamma_{i}\right)$, and hence $F(\Gamma)=$ $\cap_{i=1}^{m} F\left(\Gamma_{i}\right)$. The proof is complete.

Theorem 8. Let $C$ be a nonempty closed convex subset of a real reflexive Banach space $E$ which has a uniformly Gateaux differentiable norm. Let $T_{i}: C \rightarrow C, i=1,2, \ldots, m$, be a finite family of continuous strictly pseudocontractive mappings, let $A_{i}: C \rightarrow E^{*}, i=1,2, \ldots, m$, be a finite family of continuous monotone mappings such that $F=F_{1} \cap F_{2} \neq \emptyset$, and let $f: C \rightarrow C$ be a contraction with a contraction coefficient $\rho \in(0,1) . T_{i r_{n}}$ and $F_{i r_{n}}$ are defined as (19) and (20), respectively. Let $\left\{x_{n}\right\}$ be a sequence generated by $x_{0} \in C$

$$
\begin{gathered}
y_{n}=\lambda_{n} x_{n}+\left(1-\lambda_{n}\right) \sum_{i=1}^{m} \mu_{i} F_{i r_{n}} x_{n}, \\
x_{n+1}=\alpha_{n} f\left(x_{n}\right)+\beta_{n} x_{n}+\gamma_{n} \sum_{i=1}^{m} \sigma_{i} T_{i r_{n}} y_{n},
\end{gathered}
$$

where $\lambda_{n} \in[0,1]$ and $\left\{\alpha_{n}\right\},\left\{\beta_{n}\right\}$, and $\left\{\gamma_{n}\right\}$ are sequences of nonnegative real numbers in $[0,1]$, and

(i) $\alpha_{n}+\beta_{n}+\gamma_{n}=1, n \geq 0, \sum_{i=1}^{m} \mu_{i}=1, \sum_{i=1}^{m} \sigma_{i}=1, \mu_{i} \geq 0$, $\sigma_{i} \geq 0$;

(ii) $\lim _{n \rightarrow \infty} \alpha_{n}=0, \sum_{n=1}^{\infty} \alpha_{n}=\infty$;

(iii) $0<\lim \inf _{n \rightarrow \infty} \beta_{n}<\lim \sup _{n \rightarrow \infty} \beta_{n}<1$;

(iv) $\lim \sup _{n \rightarrow \infty} r_{n}>0, \sum_{n=1}^{\infty}\left|r_{n+1}-r_{n}\right|<\infty$.

Then the sequence $\left\{x_{n}\right\}$ converges strongly to an element $\bar{x}=$ $\Pi_{F} f(\bar{x})$, and also $\bar{x}$ is the unique solution of the variational inequality

$$
\langle(f-I)(\bar{x}), j(y-\bar{x})\rangle \leq 0, \quad \forall y \in F .
$$

Proof. First we prove that $\left\{x_{n}\right\}$ is bounded. Take $p \in F$, because $F_{i r_{n}}$ is non-expansive; then we have that

$$
\begin{aligned}
\left\|y_{n}-p\right\| \leq & \lambda_{n}\left\|x_{n}-p\right\|+\left(1-\lambda_{n}\right) \\
& \times \sum_{i=1}^{m} \mu_{i}\left\|F_{i r_{n}} x_{n}-F_{i r_{n}} p\right\| \leq\left\|x_{n}-p\right\| .
\end{aligned}
$$


For $n \geq 0$, because $T_{i r_{n}}$ and $F_{i r_{n}}$ are nonexpansive and $f$ is contractive, we have from (25) that

$$
\begin{aligned}
\left\|x_{n+1}-p\right\| & \left\|\alpha_{n}\left(f\left(x_{n}\right)-p\right)+\beta_{n}\left(x_{n}-p\right)+\gamma_{n}\left(\sum_{i=1}^{m} \sigma_{i} T_{i r_{n}} y_{n}-p\right)\right\| \\
\leq & \alpha_{n}\left\|f\left(x_{n}\right)-f(p)\right\|+\alpha_{n}\|f(p)-p\| \\
& +\beta_{n}\left\|x_{n}-p\right\|+\gamma_{n}\left\|y_{n}-p\right\| \\
\leq & \rho \alpha_{n}\left\|x_{n}-p\right\|+\alpha_{n}\|f(p)-p\|+\left(1-\alpha_{n}\right)\left\|x_{n}-p\right\| \\
\leq & {\left[1-(1-\rho) \alpha_{n}\right]\left\|x_{n}-p\right\|+\alpha_{n}\|f(p)-p\| } \\
\leq & \max \left\{\left\|x_{0}-p\right\|, \frac{f(p)-p}{1-\rho}\right\} .
\end{aligned}
$$

Therefore, $\left\{x_{n}\right\}$ is bounded. Consequently, we get that $\left\{F_{i r_{n}} x_{n}\right\},\left\{T_{i r_{n}} y_{n}\right\}$ and $\left\{y_{n}\right\},\left\{f\left(x_{n}\right)\right\}$ are bounded.

Next, we show that $\left\|x_{n+1}-x_{n}\right\| \rightarrow 0$.

\section{Consider}

$$
\begin{aligned}
& \left\|y_{n+1}-y_{n}\right\| \\
& \leq \lambda_{n+1}\left\|x_{n+1}-x_{n}\right\|+\left(1-\lambda_{n+1}\right) \sum_{i=1}^{m} \mu_{i}\left\|F_{i r_{n+1}} x_{n+1}-F_{i r_{n}} x_{n}\right\| \\
& \quad+\left|\lambda_{n+1}-\lambda_{n}\right|\left\|x_{n}-\sum_{i=1}^{m} \mu_{i} F_{i r_{n}} x_{n}\right\| .
\end{aligned}
$$

Let $v_{i n}=F_{i r_{n}} x_{n}, v_{i, n+1}=F_{i r_{n+1}} x_{n+1}$; by the definition of mapping $F_{i r_{n}}$, we have that

$$
\begin{aligned}
& \left\langle y-v_{i n}, A_{i} v_{i n}\right\rangle+\frac{1}{r_{n}}\left\langle y-v_{i n}, J v_{i n}-J x_{n}\right\rangle \geq 0, \quad \forall y \in C \\
& \left\langle y-v_{i, n+1}, A_{i} v_{i n+1}\right\rangle+\frac{1}{r_{n+1}}\left\langle y-v_{i, n+1}, J v_{i, n+1}-J x_{n+1}\right\rangle \geq 0, \\
& \left\langle v_{i, n+1}-v_{i n}, A_{i} v_{i n}\right\rangle+\frac{1}{r_{n}}\left\langle v_{i, n+1}-v_{i n}, J v_{i n}-J x_{n}\right\rangle \geq 0, \\
& \left\langle v_{i n}-v_{i, n+1}, A_{i} v_{i, n+1}\right\rangle \\
& +\frac{1}{r_{n+1}}\left\langle v_{i n}-v_{i, n+1}, J v_{i, n+1}-J x_{n+1}\right\rangle \geq 0 .
\end{aligned}
$$$$
\forall y \in C \text {. }
$$

Adding (30), we have that

$$
\begin{aligned}
& \left\langle v_{i, n+1}-v_{i n}, A_{i} v_{i n}-A_{i} v_{i, n+1}\right\rangle \\
& +\left\langle v_{i, n+1}-v_{i n}, \frac{J v_{i n}-J x_{n}}{r_{n}}-\frac{J v_{i, n+1}-J x_{n+1}}{r_{n+1}}\right\rangle \geq 0 .
\end{aligned}
$$

Since $A_{i}, i \in\{1,2, \ldots, m\}$ are monotone mappings, which implies that

$$
\left\langle v_{i, n+1}-v_{i n}, \frac{J v_{i n}-J x_{n}}{r_{n}}-\frac{J v_{i, n+1}-J x_{n+1}}{r_{n+1}}\right\rangle \geq 0,
$$

therefore we have that

$$
\begin{aligned}
& \left\langle v_{i, n+1}-v_{i n}, J v_{i n}-J x_{n}-\frac{r_{n}\left(J v_{i, n+1}-J x_{n+1}\right)}{r_{n+1}}\right. \\
& \left.\quad+J v_{i, n+1}-J v_{i, n+1}\right\rangle \geq 0 .
\end{aligned}
$$

That is,

$$
\begin{aligned}
& \left\|v_{i, n+1}-v_{i n}\right\|^{2} \\
& \leq\left\langle v_{i, n+1}-v_{i n}, J x_{n+1}-J x_{n}+\left(1-\frac{r_{n}}{r_{n+1}}\right)\right. \\
& \left.\quad \times\left(J v_{i, n+1}-J x_{n+1}\right)\right\rangle \\
& \leq\left\|v_{i, n+1}-v_{i n}\right\|\left\{\left\|x_{n+1}-x_{n}\right\|\right. \\
& \left.\quad+\left|1-\frac{r_{n}}{r_{n+1}}\right|\left\|v_{i, n+1}-x_{n+1}\right\|\right\} .
\end{aligned}
$$

Without loss of generality, let $b$ be a real number such that $r_{n}>b>0$; then we have that

$$
\begin{aligned}
\left\|v_{i, n+1}-v_{i n}\right\| & \leq\left\|x_{n+1}-x_{n}\right\|+\left|1-\frac{r_{n}}{r_{n+1}}\right|\left\|v_{i, n+1}-x_{n+1}\right\| \\
& \leq\left\|x_{n+1}-x_{n}\right\|+\frac{1}{b}\left|r_{n+1}-r_{n}\right| K,
\end{aligned}
$$

where $K=\sup \left\{\left\|v_{i, n+1}-x_{n+1}\right\|\right\}$.

Then we have from (35) and (27) that

$$
\begin{aligned}
\left\|y_{n+1}-y_{n}\right\| \leq & \left\|x_{n+1}-x_{n}\right\|+\frac{\left(1-\lambda_{n+1}\right)\left|r_{n+1}-r_{n}\right|}{b} K \\
& +\left|\lambda_{n+1}-\lambda_{n}\right|\left\|x_{n}-\sum_{i=1}^{m} \mu_{i} F_{i r_{n}} x_{n}\right\| .
\end{aligned}
$$

On the other hand, let $u_{i n}=T_{i r_{n}} y_{n}, u_{i, n+1}=T_{i r_{n+1}} y_{n+1}$; we have that

$$
\begin{array}{r}
\left\langle y-u_{i n}, T_{i} u_{i n}\right\rangle-\frac{1}{r_{n}}\left\langle y-u_{i n},\left(1+r_{n}\right) J u_{i n}-J y_{n}\right\rangle \leq 0, \\
\forall y \in C, \\
\left\langle y-u_{i, n+1}, T_{i} u_{i, n+1}\right\rangle-\frac{1}{r_{n+1}}\left\langle y-u_{i, n+1},\left(1+r_{n+1}\right)\right. \\
\left.\times J u_{i, n+1}-J y_{n+1}\right\rangle \leq 0, \\
\forall y \in C .
\end{array}
$$



that

Let $y:=u_{i, n+1}$ in (37), and let $y:=u_{\text {in }}$ in (38); we have

$$
\begin{gathered}
\left\langle u_{i, n+1}-u_{i n}, T_{i} u_{i n}\right\rangle-\frac{1}{r_{n}}\left\langle u_{i, n+1}-u_{i n},\left(1+r_{n}\right) J u_{i n}-J y_{n}\right\rangle \leq 0, \\
\left\langle u_{i n}-u_{i, n+1}, T_{i} u_{i, n+1}\right\rangle \\
-\frac{1}{r_{n+1}}\left\langle u_{i n}-u_{i, n+1},\left(1+r_{n+1}\right) J u_{i, n+1}-J y_{n+1}\right\rangle \leq 0 .
\end{gathered}
$$

Adding (39) and because $T_{i}, i \in\{1,2, \ldots, m\}$ is pseudocontractive, we have that

$$
\left\langle u_{i, n+1}-u_{i n}, \frac{J u_{i n}-J y_{n}}{r_{n}}-\frac{J u_{i, n+1}-J y_{n+1}}{r_{n+1}}\right\rangle \geq 0 .
$$

Therefore we have

$$
\begin{aligned}
& \left\langle u_{i, n+1}-u_{i n}, J u_{i n}-J y_{n}-\frac{r_{n}\left(J u_{i, n+1}-J y_{n+1}\right)}{r_{n+1}}\right. \\
& \left.+J u_{i, n+1}-J u_{i, n+1}\right\rangle \geq 0 .
\end{aligned}
$$

Hence we have that

$$
\left\|u_{i, n+1}-u_{i n}\right\| \leq\left\|y_{n+1}-y_{n}\right\|+\frac{1}{b}\left|r_{n+1}-r_{n}\right| M
$$

where $M=\sup \left\{\left\|u_{i n}-y_{n}\right\|\right\}$.

Let $x_{n+1}=\beta_{n} x_{n}+\left(1-\beta_{n}\right) z_{n}$. Hence we have that

$$
\begin{aligned}
z_{n+1}- & z_{n} \\
= & \frac{\alpha_{n+1}}{1-\beta_{n+1}}\left(f\left(x_{n+1}\right)-f\left(x_{n}\right)\right) \\
& +\left(\frac{\alpha_{n+1}}{1-\beta_{n+1}}-\frac{\alpha_{n}}{1-\beta_{n}}\right) f\left(x_{n}\right) \\
& +\frac{\gamma_{n+1}}{1-\beta_{n+1}}\left(u_{i, n+1}-u_{i n}\right) \\
& +\left(\frac{\gamma_{n+1}}{1-\beta_{n+1}}-\frac{\gamma_{n}}{1-\beta_{n}}\right) u_{i n} .
\end{aligned}
$$

Hence we have from (43), (42), and (36) that

$$
\begin{aligned}
& \left\|z_{n+1}-z_{n}\right\|-\left\|x_{n+1}-x_{n}\right\| \\
& \leq \frac{(\rho-1) \alpha_{n+1}}{1-\beta_{n+1}}\left\|x_{n+1}-x_{n}\right\|+\left|\frac{\alpha_{n+1}}{1-\beta_{n+1}}-\frac{\alpha_{n}}{1-\beta_{n}}\right| \\
& \quad \times\left\{\left\|f\left(x_{n}\right)\right\|+\left\|u_{i n}\right\|\right\}+\frac{\gamma_{n+1}}{1-\beta_{n+1}} \frac{\left|r_{n+1}-r_{n}\right|}{b} \\
& \quad \times\left(\left(1-\lambda_{n+1}\right) K+M\right)+\frac{\gamma_{n+1}}{1-\beta_{n+1}}\left|\lambda_{n+1}-\lambda_{n}\right| \\
& \quad \times\left\|x_{n}-\sum_{i=1}^{m} \mu_{i} F_{i r_{n}} x_{n}\right\| .
\end{aligned}
$$

Noticing the conditions (ii) and (iv), we have that

$$
\limsup _{n \rightarrow \infty}\left(\left\|z_{n+1}-z_{n}\right\|-\left\|x_{n+1}-x_{n}\right\|\right)=0 .
$$

Hence we have from Lemma 4 that

$$
\limsup _{n \rightarrow \infty}\left\|z_{n}-x_{n}\right\|=0 \text {. }
$$

Therefore we have that

$$
\left\|x_{n+1}-x_{n}\right\|=\left|1-\beta_{n}\right|\left\|z_{n}-x_{n}\right\| \longrightarrow 0 .
$$

Hence we have from (35), (36), and (42) that

$$
\begin{gathered}
\left\|y_{n+1}-y_{n}\right\| \longrightarrow 0, \\
\left\|u_{i, n+1}-u_{i n}\right\| \longrightarrow 0, \\
\left\|v_{i, n+1}-v_{i n}\right\| \longrightarrow 0 .
\end{gathered}
$$

In addition, since $x_{n+1}=\alpha_{n} f\left(x_{n}\right)+\beta_{n} x_{n}+\gamma_{n} \sum_{i=1}^{m} \sigma_{i} u_{i n}$, $y_{n}=\lambda_{n} x_{n}+\left(1-\lambda_{n}\right) \sum_{i=1}^{m} \mu_{i} v_{i n}$, for all $p \in F$, we have from the monotonicity of $A_{i}$, the non-expansivity of $T_{i r_{n}}$, and the convexity of $\|\cdot\|^{2}$ that

$$
\begin{aligned}
\left\|x_{n+1}-p\right\|^{2} & \left\|\alpha_{n} f\left(x_{n}\right)+\beta_{n} x_{n}+\gamma_{n} \sum_{i=1}^{m} \sigma_{i} u_{i n}-p\right\|^{2} \\
\leq & \left\|\alpha_{n}\left(f\left(x_{n}\right)-p\right)+\beta_{n}\left(x_{n}-p\right)\right\|^{2}+\gamma_{n} \sum_{i=1}^{m} \sigma_{i}\left\|u_{i n}-p\right\|^{2} \\
\leq & \alpha_{n}\left\|f\left(x_{n}\right)-p\right\|^{2}+\beta_{n}\left\|x_{n}-p\right\|^{2}+\gamma_{n}\left\|y_{n}-p\right\|^{2} \\
\leq & \alpha_{n}\left\|f\left(x_{n}\right)-p\right\|^{2}+\beta_{n}\left\|x_{n}-p\right\|^{2} \\
& +\gamma_{n} \lambda_{n}\left\|x_{n}-p\right\|^{2}+\left(1-\lambda_{n}\right) \gamma_{n}\left\|v_{n}-p\right\|^{2} \\
\leq & \alpha_{n}\left\|f\left(x_{n}\right)-p\right\|^{2}+\left(\beta_{n}+\gamma_{n} \lambda_{n}\right)\left\|x_{n}-p\right\|^{2} \\
& +\left(1-\lambda_{n}\right) \gamma_{n}\left(\left\|x_{n}-p\right\|^{2}-\left\|x_{n}-v_{i n}\right\|^{2}\right) \\
\leq & \alpha_{n}\left\|f\left(x_{n}\right)-p\right\|^{2}+\left\|x_{n}-p\right\|^{2}-\left(1-\lambda_{n}\right) \gamma_{n}\left\|x_{n}-v_{i n}\right\|^{2} .
\end{aligned}
$$

So we have that

$$
\begin{aligned}
& \left(1-\lambda_{n}\right) \gamma_{n}\left\|x_{n}-v_{i n}\right\|^{2} \\
& \leq \alpha_{n}\left\|f\left(x_{n}\right)-p\right\|^{2}+\left\|x_{n}-p\right\|^{2}-\left\|x_{n+1}-p\right\|^{2} \\
& \leq \alpha_{n}\left\|f\left(x_{n}\right)-p\right\|^{2}+\left\|x_{n}-x_{n+1}\right\| \times\left(\left\|x_{n}-p\right\|+\left\|x_{n+1}-p\right\|\right) .
\end{aligned}
$$

Since $\alpha_{n} \rightarrow 0$, so we have from (47) that

$$
\left\|x_{n}-v_{\text {in }}\right\| \longrightarrow 0 \text {. }
$$

In a similar way, we have that

$$
\left\|x_{n}-u_{i n}\right\| \longrightarrow 0 .
$$


Consequently, we have that

$$
\begin{gathered}
\left\|y_{n}-x_{n}\right\|=\left|1-\lambda_{n}\right|\left\|x_{n}-v_{i n}\right\| \longrightarrow 0, \\
\left\|y_{n}-u_{i n}\right\| \leq\left\|y_{n}-x_{n}\right\|+\left\|x_{n}-u_{i n}\right\| \longrightarrow 0 .
\end{gathered}
$$

Since $E$ is a reflexive real Banach space and the sequence $\left\{x_{n}\right\}$ is bounded, so there exists the subsequence $\left\{x_{n k}\right\}$ of $\left\{x_{n}\right\}$ and $w \in C$ such that $x_{n k} \rightarrow w$. And because $x_{n} \rightarrow v_{i n}$, $n \rightarrow \infty$, therefore $v_{\text {ink }} \rightarrow w$. Next we show that $w \in F$.

Because $v_{i n}=F_{i r_{n}} x_{n}$, by the definition of mapping $F_{i r_{n}}$, we have that

$$
\begin{gathered}
\left\langle y-v_{i n}, A_{i} v_{i n}\right\rangle+\frac{1}{r_{n}}\left\langle y-v_{i n}, J v_{i n}-J x_{n}\right\rangle \geq 0, \quad \forall y \in C, \\
\left\langle y-v_{i n k}, A_{i} v_{i n k}\right\rangle+\left\langle y-v_{i n}, \frac{J v_{i n k}-J x_{n k}}{r_{n}}\right\rangle \geq 0, \quad \forall y \in C .
\end{gathered}
$$

Let $v_{t}=t v+(1-t) w, t \in[0,1]$, for all $v \in C$; we have that

$$
\begin{aligned}
\left\langle v_{t}-v_{i n k}, A_{i} v_{t}\right\rangle \geq & \left\langle v_{t}-v_{i n k}, A_{i} v_{t}\right\rangle-\left\langle v_{t}-v_{i n k}, A_{i} v_{i n k}\right\rangle \\
& -\left\langle v_{t}-v_{i n k}, \frac{J v_{i n k}-J x_{n k}}{r_{n}}\right\rangle \\
= & \left\langle v_{t}-v_{i n k}, A_{i} v_{t}-A_{i} v_{i n k}\right\rangle \\
& -\left\langle v_{t}-v_{i n k}, \frac{J v_{i n k}-J x_{n k}}{r_{n}}\right\rangle .
\end{aligned}
$$

Because $x_{n k}-v_{i n k} \rightarrow 0$, so $\left(J v_{i n k}-J x_{n k}\right) / r_{n} \rightarrow 0$, and because $A_{i}$ are monotone, we have that

$$
0 \leq \lim _{k \rightarrow \infty}\left\langle v_{t}-v_{i n k}, A_{i} v_{t}\right\rangle=\left\langle v_{t}-w, A_{i} v_{t}\right\rangle
$$

Consequently we have that

$$
\left\langle v-w, A_{i} v_{t}\right\rangle \geq 0
$$

If $t \rightarrow 0$, by the continuity of $A_{i}$, we have that $\langle v-$ $\left.w, A_{i} w\right\rangle \geq 0$; that is, $w \in V I\left(C, A_{i}\right)$, and then $w \in F_{2}$.

Similarly, because $u_{i n}=T_{i r_{n}} y_{n}$, by the definition of mapping $T_{i r_{n}}$, we have that

$$
\begin{array}{r}
\left\langle y-u_{i n}, T_{i} u_{i n}\right\rangle-\frac{1}{r_{n}}\left\langle y-u_{i n},\left(1+r_{n}\right) J u_{i n}-J y_{n}\right\rangle \leq 0, \\
\forall y \in C, \\
\left\langle y-u_{i n k}, T_{i} u_{i n k}\right\rangle-\frac{1}{r_{n}}\left\langle y-u_{i n k},\left(1+r_{n}\right) J u_{i n k}-J y_{n k}\right\rangle \leq 0, \\
\forall y \in C .
\end{array}
$$

Let $v_{t}=t v+(1-t) w, t \in[0,1]$, for all $v \in C$. Because $\left\{T_{i}\right\}, i \in\{1,2, \ldots, m\}$ are pseudocontractive mappings, we have that

$$
\begin{aligned}
\left\langle u_{i n k}-v_{t}, T_{i} v_{t}\right\rangle & \left.\geq u_{i n k}-v_{t}, T_{i} v_{t}\right\rangle+\left\langle v_{t}-u_{i n k}, T_{i} u_{i n k}\right\rangle \\
& -\frac{1}{r_{n}}\left\langle v_{t}-u_{i n k},\left(1+r_{n}\right) J u_{i n k}-J y_{n k}\right\rangle \\
= & \left\langle v_{t}-u_{i n k}, T_{i} u_{i n k}-T_{i} v_{t}\right\rangle \\
& -\left\langle v_{t}-u_{i n k}, \frac{1+r_{n}}{r_{n}} J u_{i n k}-\frac{1}{r_{n}} J y_{n k}\right\rangle \\
\geq & -\left\|v_{t}-u_{i n k}\right\|^{2}-\frac{1}{r_{n}}\left\langle v_{t}-u_{i n k}, J u_{i n k}-J y_{n k}\right\rangle \\
& -\left\langle v_{t}-u_{i n k}, J u_{i n k}\right\rangle \\
= & -\left\langle v_{t}-u_{i n k}, J v_{t}\right\rangle-\frac{1}{r_{n}}\left\langle v_{t}-u_{i n k}, J u_{i n k}-J y_{n k}\right\rangle .
\end{aligned}
$$
that

Because $y_{n k}-u_{i n k} \rightarrow 0$, so $J u_{i n k}-J y_{n k} \rightarrow 0$; we have

$$
\lim _{k \rightarrow \infty}\left\langle u_{i n k}-v_{t}, T_{i} v_{t}\right\rangle \geq \lim _{k \rightarrow \infty}\left\langle u_{\text {ink }}-v_{t}, J v_{t}\right\rangle .
$$

Consequently we have that

$$
\begin{gathered}
\left\langle w-v_{t}, T_{i} v_{t}\right\rangle \geq\left\langle w-v_{t}, J v_{t}\right\rangle, \\
\left\langle v-w, T_{i} v_{t}\right\rangle \leq\left\langle v-w, J v_{t}\right\rangle .
\end{gathered}
$$

If $t \rightarrow 0$, by the continuity of $T_{i}$, we have that $\left\langle v-w, T_{i} w-\right.$ $J w\rangle \geq 0$, for all $v \in C$; we conclude that $w=T_{i} w$; that is, $w \in F\left(T_{i}\right)$, and then $w \in F_{1}$. Consequently $w \in F=F_{1} \cap F_{2}$.

Because $\bar{x}=\Pi_{F} f(\bar{x})$, we have from Lemma 5 that

$$
\begin{array}{r}
\limsup _{n \rightarrow \infty}\left\langle f(\bar{x})-\bar{x}, j\left(x_{n+1}-\bar{x}\right)\right\rangle \\
=\langle f(\bar{x})-\bar{x}, j(w-\bar{x})\rangle \leq 0 .
\end{array}
$$

Next we show that $x_{n} \rightarrow \bar{x}$. Since $u_{i n}=T_{i r_{n}} y_{n}$, from formula (23) and Lemma 6 we have that

$$
\begin{aligned}
& \left\|x_{n+1}-\bar{x}\right\|^{2} \\
& =\left\|\alpha_{n}\left(f\left(x_{n}\right)-\bar{x}\right)+\beta_{n}\left(x_{n}-\bar{x}\right)+\gamma_{n}\left(\sum_{i=1}^{m} \sigma_{i} T_{i r_{n}} y_{n}-\bar{x}\right)\right\|^{2} \\
& \leq\left\|\beta_{n}\left(x_{n}-\bar{x}\right)+\gamma_{n}\left(\sum_{i=1}^{m} \sigma_{i} u_{i n}-\bar{x}\right)\right\|^{2} \\
& \quad+2 \alpha_{n}\left\langle f\left(x_{n}\right)-\bar{x}, j\left(x_{n+1}-\bar{x}\right)\right\rangle
\end{aligned}
$$




$$
\begin{aligned}
\leq & \left(1-\alpha_{n}\right)^{2}\left\|x_{n}-\bar{x}\right\|^{2}+2 \alpha_{n}\left\langle f\left(x_{n}\right)-f(\bar{x}), j\left(x_{n+1}-\bar{x}\right)\right\rangle \\
& +2 \alpha_{n}\left\langle f(\bar{x})-\bar{x}, j\left(x_{n+1}-\bar{x}\right)\right\rangle \\
\leq & \left(1-\alpha_{n}\right)^{2}\left\|x_{n}-\bar{x}\right\|^{2}+2 \rho \alpha_{n}\left\|x_{n}-\bar{x}\right\|\left\|x_{n+1}-\bar{x}\right\| \\
& +2 \alpha_{n}\left\langle f(\bar{x})-\bar{x}, j\left(x_{n+1}-\bar{x}\right)\right\rangle \\
\leq & \left(1-2 \alpha_{n}(1-\rho)\right)\left\|x_{n}-\bar{x}\right\|^{2}+\sigma_{n}
\end{aligned}
$$

where $M_{1}=\sup \left\{\left\|x_{n}-\bar{x}\right\|\right\}, \sigma_{n}=\alpha_{n}^{2} M_{1}^{2}+2 \rho \alpha_{n}\left\|x_{n+1}-x_{n}\right\| M_{1}+$ $2 \alpha_{n}\left\langle f(\bar{x})-\bar{x}, j\left(x_{n+1}-\bar{x}\right)\right\rangle$. Let $\theta_{n}=2 \alpha_{n}(1-\rho)$; according to Lemma 1 and formula (62), we have that $\lim _{n \rightarrow \infty}\left\|x_{n}-\bar{x}\right\|=0$; that is, the sequence $\left\{x_{n}\right\}$ converges strongly to $\bar{x} \in F$.

According to formula (62) we conclude that $\bar{x}$ is the solution of the variational inequality (24). Now we show that $\bar{x}$ is the unique solution of the variational inequality (24). Suppose that $\bar{y} \in F$ is another solution of the variational inequality (24). Because $\bar{x}$ is the solution of the variational inequality (24), that is, $\langle(f-I) \bar{x}, j(y-\bar{x})\rangle \leq 0$, for all $y \in F$. And because that $\bar{y} \in F$, then we have

$$
\langle(f-I) \bar{x}, j(\bar{y}-\bar{x})\rangle \leq 0 .
$$

On the other hand, to the solution $\bar{y} \in F$, since $\bar{x} \in F$, so

$$
\langle(f-I) \bar{y}, j(\bar{x}-\bar{y})\rangle \leq 0 .
$$

Adding (65) and (64), we have that

$$
\langle\bar{x}-\bar{y}-(f(\bar{x})-f(\bar{y})), j(\bar{x}-\bar{y})\rangle \leq 0 .
$$

Hence

$$
(1-\rho)\|\bar{x}-\bar{y}\|^{2} \leq 0 .
$$

Because $\rho \in(0,1)$, hence we conclude that $\bar{x}=\bar{y}$, and the uniqueness of the solution is obtained.

Theorem 9. Let $C$ be a nonempty closed convex subset of a real reflexive Banach space $E$ which has a uniformly Gateaux differentiable norm. Let $T_{i}: C \rightarrow C, i=1,2, \ldots, m$, be a finite family of continuous strictly pseudocontractive mappings, let $A_{i}: C \rightarrow E^{*}, i=1,2, \ldots, m$, be a finite family of continuous monotone mappings such that $F=F_{1} \cap F_{2} \neq \emptyset$, and let $f: C \rightarrow C$ be a contraction with a contraction coefficient $\rho \in(0,1) . T_{i r_{n}}$ and $F_{i r_{n}}$ are defined as (19) and (20), respectively. Let $x_{n}$ be a sequence generated by $x_{0} \in C$

$$
x_{n+1}=\alpha_{n} f\left(x_{n}\right)+\beta_{n} x_{n}+\gamma_{n} \sum_{i=1}^{m} \varepsilon_{i} T_{i r_{n}} F_{i r_{n}} x_{n},
$$

where $\left\{\alpha_{n}\right\},\left\{\beta_{n}\right\}$, and $\left\{\gamma_{n}\right\}$ are sequences of nonnegative real numbers in $[0,1]$, and

(i) $\alpha_{n}+\beta_{n}+\gamma_{n}=1, n \geq 0, \sum_{i=1}^{m} \varepsilon_{i}=1, \varepsilon_{i} \geq 0$;

(ii) $\lim _{n \rightarrow \infty} \alpha_{n}=0, \sum_{n=1}^{\infty} \alpha_{n}=\infty$;

(iii) $0<\lim \inf _{n \rightarrow \infty} \beta_{n}<\lim \sup _{n \rightarrow \infty} \beta_{n}<1$;

(iv) $\lim \sup _{n \rightarrow \infty} r_{n}>0, \sum_{n=1}^{\infty}\left|r_{n+1}-r_{n}\right|<\infty$.
Then the sequence $x_{n}$ converges strongly to an element $\bar{x}=$ $\Pi_{F} f(\bar{x})$, and also $\bar{x}$ is the unique solution of the variational inequality

$$
\langle(f-I)(\bar{x}), j(y-\bar{x})\rangle \leq 0, \quad \forall y \in F .
$$

Proof. Putting $\lambda_{n}=0$ in Theorem 8, we can obtain the result.

If, in Theorems 8 and 9 , we let $f: \equiv u \in C$ be a constant mapping, we have the following corollaries.

Corollary 10. Let $C$ be a nonempty closed convex subset of a real reflexive Banach space $E$ which has a uniformly Gateaux differentiable norm. Let $T_{i}: C \rightarrow C, i=1,2, \ldots, m$, be a finite family of continuous strictly pseudo-contractive mappings, and $A_{i}: C \rightarrow E^{*}, i=1,2, \ldots, m$, be a finite family of continuous monotone mappings such that $F=F_{1} \cap F_{2} \neq \emptyset . T_{i r_{n}}$ and $F_{i r_{n}}$ are defined as (19) and (20), respectively. Let $x_{n}$ be a sequence generated by $x_{0} \in C$

$$
\begin{gathered}
y_{n}=\lambda_{n} x_{n}+\left(1-\lambda_{n}\right) \sum_{i=1}^{m} \mu_{i} F_{i r_{n}} x_{n}, \\
x_{n+1}=\alpha_{n} u+\beta_{n} x_{n}+\gamma_{n} \sum_{i=1}^{m} \sigma_{i} T_{i r_{n}} y_{n},
\end{gathered}
$$

where $\lambda_{n} \in[0,1]$ and $\left\{\alpha_{n}\right\},\left\{\beta_{n}\right\}$, and $\left\{\gamma_{n}\right\}$ are sequences of nonnegative real numbers in $[0,1]$, and

(i) $\alpha_{n}+\beta_{n}+\gamma_{n}=1, n \geq 0, \sum_{i=1}^{m} \mu_{i}=1, \sum_{i=1}^{m} \sigma_{i}=1, \mu_{i} \geq 0$, $\sigma_{i} \geq 0$

(ii) $\lim _{n \rightarrow \infty} \alpha_{n}=0, \sum_{n=1}^{\infty} \alpha_{n}=\infty$;

(iii) $0<\lim \inf _{n \rightarrow \infty} \beta_{n}<\lim \sup _{n \rightarrow \infty} \beta_{n}<1$;

(iv) $\lim \sup _{n \rightarrow \infty} r_{n}>0, \sum_{n=1}^{\infty}\left|r_{n+1}-r_{n}\right|<\infty$.

Then the sequence $x_{n}$ converges strongly to $\bar{x}=\Pi_{F} u$, and also $\bar{x}$ is the unique solution of the variational inequality

$$
\langle u-\bar{x}, j(y-\bar{x})\rangle \leq 0, \quad \forall y \in F .
$$

Corollary 11. Let $C$ be a nonempty closed convex subset of a real reflexive Banach space $E$ which has a uniformly Gateaux differentiable norm. Let $T_{i}: C \rightarrow C, i=1,2, \ldots, m$, be a finite family of continuous strictly pseudocontractive mappings, and $A_{i}: C \rightarrow E^{*}, i=1,2, \ldots, m$, be a finite family of continuous monotone mappings such that $F=F_{1} \cap F_{2} \neq \emptyset . T_{i r_{n}}$ and $F_{i r_{n}}$ are defined as (19) and (20), respectively. Let $x_{n}$ be a sequence generated by $x_{0} \in C$

$$
x_{n+1}=\alpha_{n} u+\beta_{n} x_{n}+\gamma_{n} \sum_{i=1}^{m} \varepsilon_{i} T_{i r_{n}} F_{i r_{n}} x_{n},
$$

where $\left\{\alpha_{n}\right\},\left\{\beta_{n}\right\}$, and $\left\{\gamma_{n}\right\}$ are sequences of nonnegative real numbers in $[0,1]$, and

(i) $\alpha_{n}+\beta_{n}+\gamma_{n}=1, n \geq 0, \sum_{i=1}^{m} \varepsilon_{i}=1, \varepsilon_{i}>0$;

(ii) $\lim _{n \rightarrow \infty} \alpha_{n}=0, \sum_{n=1}^{\infty} \alpha_{n}=\infty$;

(iii) $0<\liminf _{n \rightarrow \infty} \beta_{n}<\lim \sup _{n \rightarrow \infty} \beta_{n}<1$;

(iv) $\lim \sup _{n \rightarrow \infty} r_{n}>0, \quad \sum_{n=1}^{\infty}\left|r_{n+1}-r_{n}\right|<\infty$. 
Then the sequence $x_{n}$ converges strongly to $\bar{x}=\Pi_{F} u$, and also $\bar{x}$ is the unique solution of the variational inequality

$$
\langle u-\bar{x}, j(y-\bar{x})\rangle \leq 0, \quad \forall y \in F .
$$

\section{Acknowledgments}

This work is supported by the National Science Foundation of China (11001287), Natural Science Foundation Project of Chongqing (CSTC, 2012jjA00039).

\section{References}

[1] B. Halpern, "Fixed points of nonexpanding maps," Bulletin of the American Mathematical Society, vol. 73, pp. 957-961, 1967.

[2] Y. Yao, "A general iterative method for a finite family of nonexpansive mappings," Nonlinear Analysis, Theory, Methods and Applications, vol. 66, no. 12, pp. 2676-2687, 2007.

[3] Y. Yao, R. Chen, and J.-C. Yao, "Strong convergence and certain control conditions for modified Mann iteration," Nonlinear Analysis, Theory, Methods and Applications, vol. 68, no. 6, pp. 1687-1693, 2008.

[4] S. S. Chang, H. W. Joseph Lee, and C. K. Chan, "On Reich's strong convergence theorem for asymptotically nonexpansive mappings in Banach spaces," Nonlinear Analysis, Theory, Methods and Applications, vol. 66, no. 11, pp. 2364-2374, 2007.

[5] H.-K. Xu, "Viscosity approximation methods for nonexpansive mappings," Journal of Mathematical Analysis and Applications, vol. 298, no. 1, pp. 279-291, 2004.

[6] S.-Y. Matsushita and W. Takahashi, "Strong convergence theorems for nonexpansive nonself-mappings without boundary conditions," Nonlinear Analysis, Theory, Methods and Applications, vol. 68, no. 2, pp. 412-419, 2008.

[7] L. Deng and Q. Liu, "Iterative scheme for nonself generalized asymptotically quasi-nonexpansive mappings," Applied Mathematics and Computation, vol. 205, no. 1, pp. 317-324, 2008.

[8] Y. Song, "A new sufficient condition for the strong convergence of Halpern type iterations," Applied Mathematics and Computation, vol. 198, no. 2, pp. 721-728, 2008.

[9] N. Shioji and W. Takahashi, "Strong convergence of approximated sequences for nonexpansive mappings in danach spaces," Proceedings of the American Mathematical Society, vol. 125, no. 12, pp. 3641-3645, 1997.

[10] W. Takahashi and K. Zembayashi, "Strong and weak convergence theorems for equilibrium problems and relatively nonexpansive mappings in Banach spaces," Nonlinear Analysis, Theory, Methods and Applications, vol. 70, no. 1, pp. 45-57, 2009.

[11] H. Zegeye, "An iterative approximation for a common fixed point of two Pseudo-contractive mappings," ISRN Mathematical Analysis, vol. 2011, Article ID 621901, 14 pages, 2011.

[12] W. Takahashi, Non-Linear Functional Analysis-Fixed Point Theory and Its Applications, Yokohama Publishers Inc, Yokohama, Japan, 2000, (in Japanese).

[13] J. Lou, L.-J. Zhang, and Z. He, "Viscosity approximation methods for asymptotically nonexpansive mappings," Applied Mathematics and Computation, vol. 203, no. 1, pp. 171-177, 2008.

[14] L.-C. Ceng, H.-K. Xu, and J.-C. Yao, "The viscosity approximation method for asymptotically nonexpansive mappings in Banach spaces," Nonlinear Analysis, Theory, Methods and Applications, vol. 69, no. 4, pp. 1402-1412, 2008.
[15] Y. Song and R. Chen, "Strong convergence theorems on an iterative method for a family of finite nonexpansive mappings," Applied Mathematics and Computation, vol. 180, no. 1, pp. 275287, 2006.

[16] H. Zegeye and N. Shahzad, "Approximating common solution of variational inequality problems for two monotone mappings in Banach spaces," Optimization Letters, vol. 5, no. 4, pp. 691$704,2011$.

[17] D.-J. Wen, "Projection methods for a generalized system of nonconvex variational inequalities with different nonlinear operators," Nonlinear Analysis, Theory, Methods and Applications, vol. 73, no. 7, pp. 2292-2297, 2010.

[18] A. Moudifi, "Viscosity approximation methods for fixed point problems," Journal of Mathematical Analysis and Applications, vol. 241, no. 1, pp. 46-55, 2000.

[19] W. Takahashi and M. Toyoda, "Weak convergence theorems for nonexpansive mappings and monotone mappings," Journal of Optimization Theory and Applications, vol. 118, no. 2, pp. 417428, 2003.

[20] W. Takahashi, Nonlinear Functional Analysis, Yokohama, Yokohama, Japan, 2000.

[21] H. Zegeye and N. Shahzad, "Strong convergence of an iterative method for pseudo-contractive and monotone mappings," Journal of Global Optimization, vol. 54, no. 1, pp. 173-184, 2011.

[22] H. Zegeye and N. Shahzad, "Strong convergence theorems for a common zero of a countably infinite family of $\alpha$-inverse strongly accretive mappings," Nonlinear Analysis, Theory, Methods and Applications, vol. 71, no. 1-2, pp. 531-538, 2009.

[23] T. Suzuki, “Strong convergence of Krasnoselskii and Mann's type sequences for one-parameter nonexpansive semigroups without Bochner integrals," Journal of Mathematical Analysis and Applications, vol. 305, no. 1, pp. 227-239, 2005. 


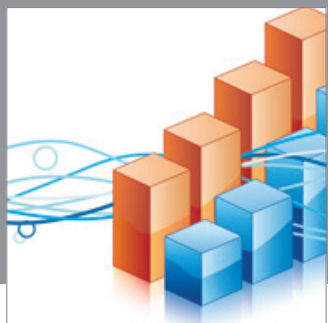

Advances in

Operations Research

mansans

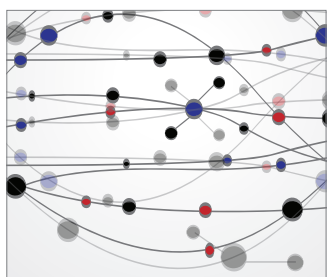

The Scientific World Journal
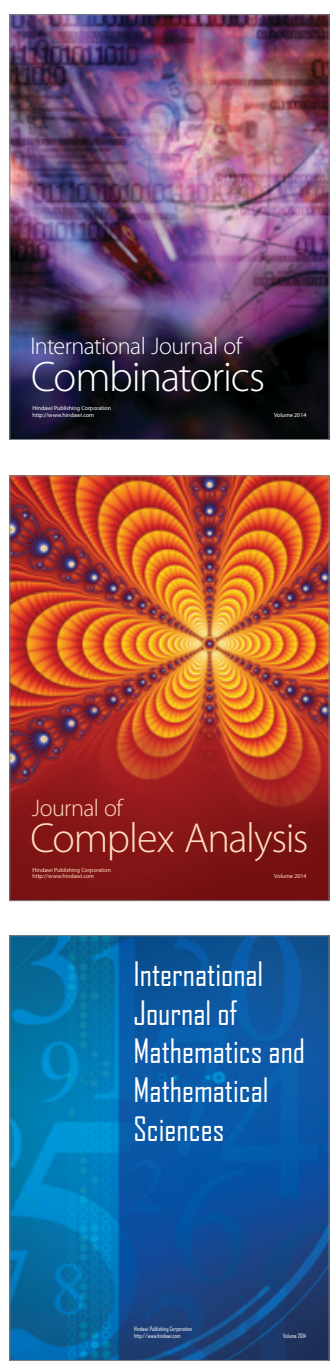
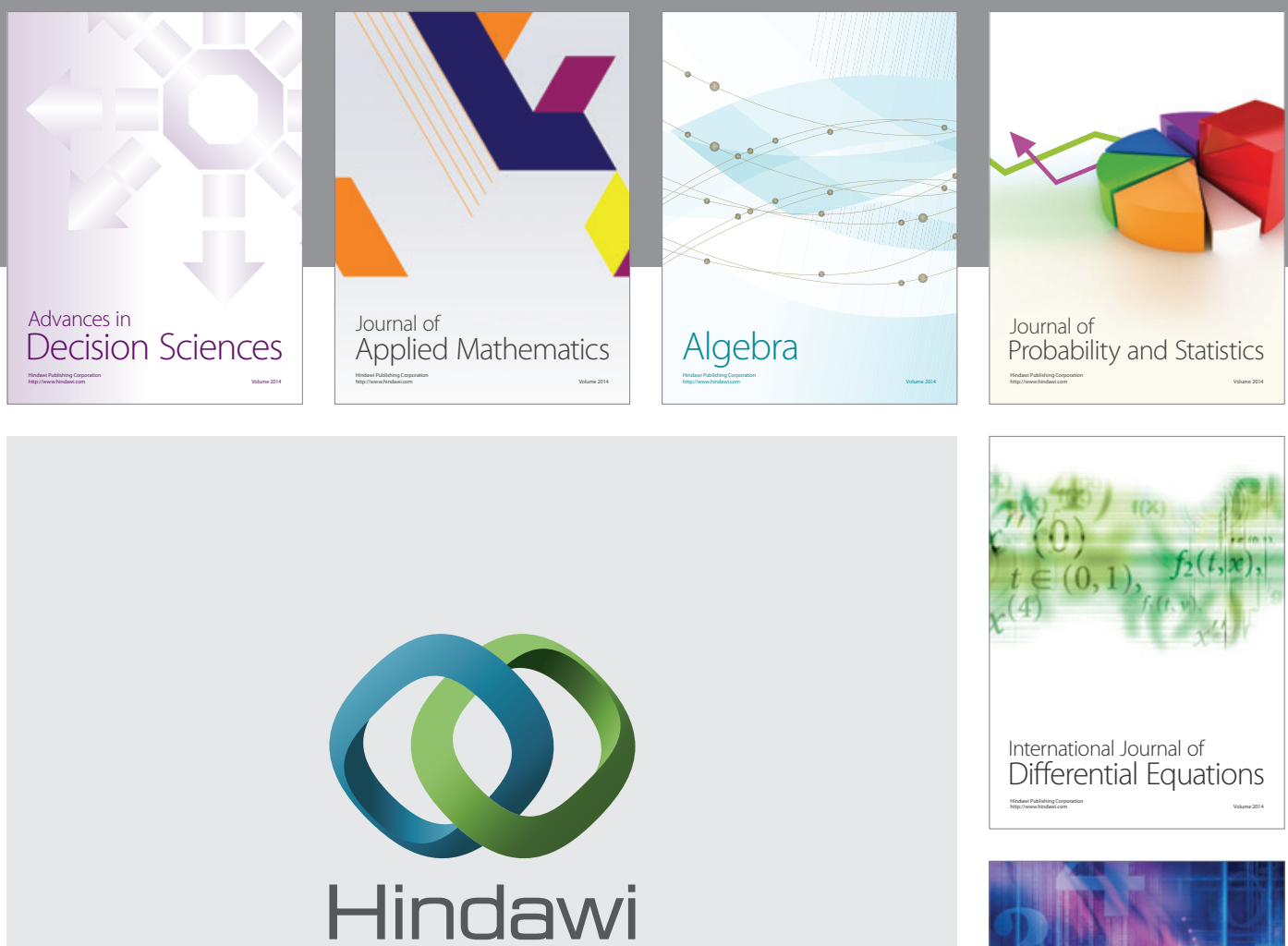

Submit your manuscripts at http://www.hindawi.com
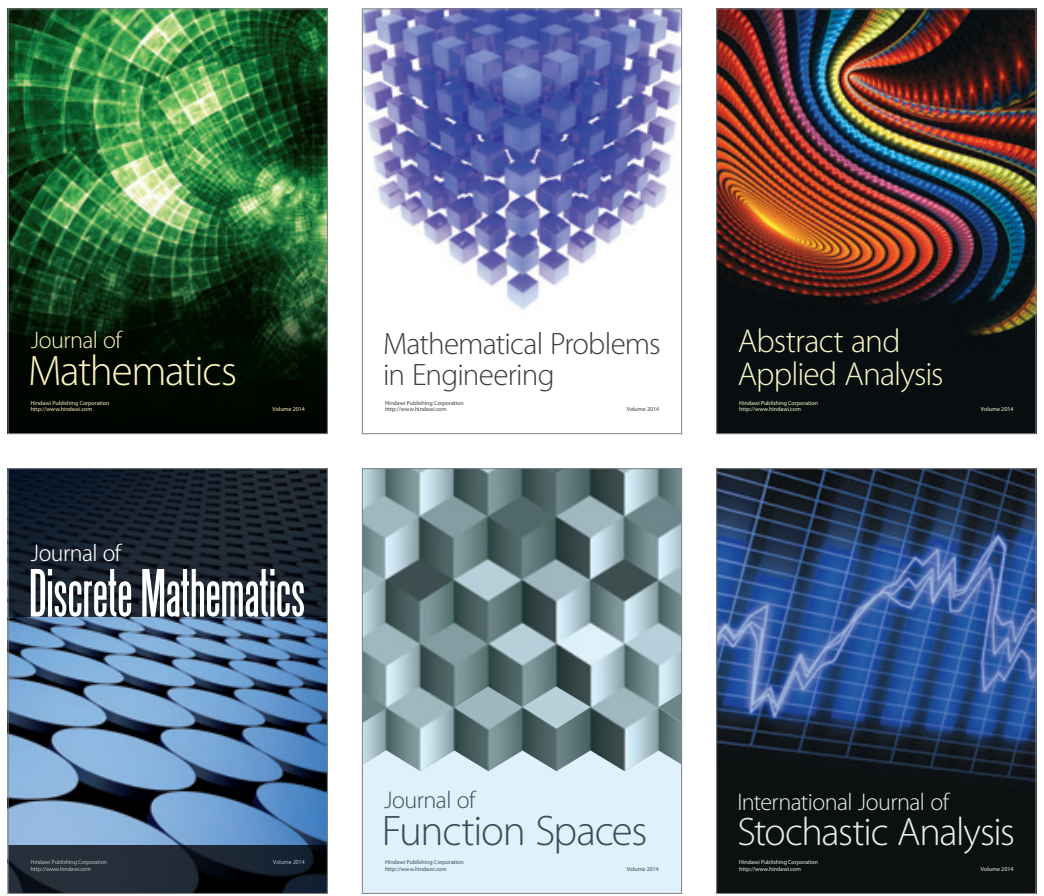

Journal of

Function Spaces

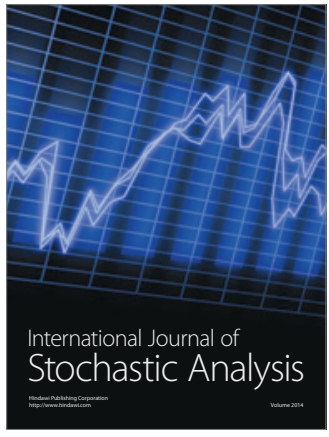

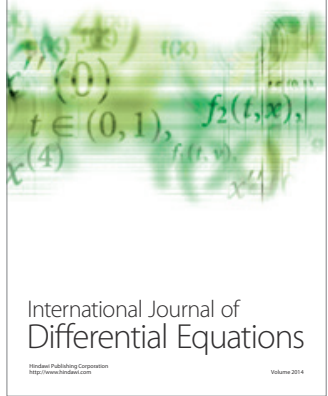
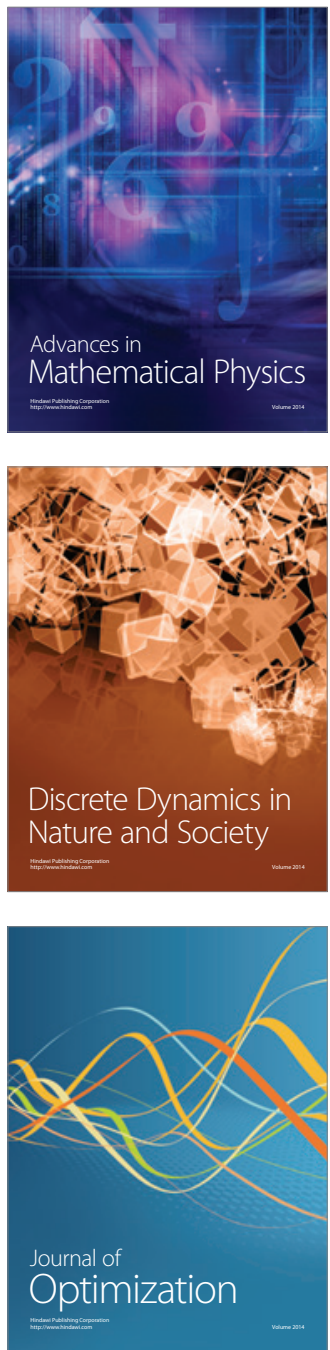